\title{
Temperature Sensitivity of InGaAs Quantum-Dot Lasers Grown by MOCVD
}

\author{
N.-H. Kim, J.-H. Park, L. J. Mawst, Senior Member, IEEE, T. F. Kuech, and M. Kanskar
}

\begin{abstract}
Temperature-dependent cavity length studies have been performed on multiple stack strain compensated InGaAs quantum-dot (QD) active region broad stripe laser structures grown by metal-organic chemical vapor deposition. The characteristic temperature coefficients of the threshold current density $\left(T_{0}\right)$ and external differential quantum efficiency $\left(T_{1}\right)$ were calculated from variable temperature measurements. The correlation of the $T_{0}, T_{1}$ values and the extracted values of the characteristic temperature coefficients of the transparency current density, material gain, injection efficiency, and internal loss $\left(T_{\mathrm{tr}}, T_{g 0}, T_{\eta \mathrm{inj}}, T_{\alpha i}\right)$ from the temperature-dependent study is discussed. The $T_{1}$ values are higher than $400 \mathrm{~K}$ for five-stack QD laser structures, comparable values to conventional quantum-well $(\mathrm{QW})$ laser structures. $T_{0}$ values are lower than $100 \mathrm{~K}$. Extracted material gain parameters are found to increase with increasing temperature for the three-stack QD structure, and are nearly temperature independent for the five-stack structure, different to that observed in InGaAs QW lasers.
\end{abstract}

Index Terms-Characteristic temperature coefficient, epitaxial growth, material gain, quantum dots (QDs), semiconductor lasers.

\section{INTRODUCTION}

$\mathbf{T}$ HE USE of a low-dimensional quantum-dot (QD) active region holds potential for realizing extremely low threshold current density lasers that are temperature insensitive [1]. Both of these factors are important for achieving high output power and high total power conversion efficiency $\eta_{p}$ in diode lasers. Ideally, the threshold current density $J_{\text {th }}$ and differential quantum efficiency $\eta_{d}$ of a QD laser should remain invariant to temperature changes. Self-assembled QDs suffer from nonuniform size distributions and the existence of excited QD and wetting layer states which prohibit realizing the full potential of an idealized system with zero-dimensional confinement. Identifying the physical mechanisms responsible for the observed temperature sensitivity of the $J_{\mathrm{th}}$ and $\eta_{d}$ in the self-assembled QD lasers is important for achieving high output power device optimization. Cavity length analysis, as presented here, can help with understanding the underlying reasons for device temperature sensitivity in QD lasers.

QD lasers with high performance have been achieved for lasing on the ground state QD transition [2]. However, there

Manuscript received August 5, 2005; revised January 4, 2006. This work was supported by the Defense Advanced Research Projects Agency (DARPA) SHEDS Project MDA972-03-9-0002.

N. H. Kim, J. H. Park, and L. J. Mawst are with the Reed Center for Photonics, Department of Electrical and Computer Engineering, University of WisconsinMadison, Madison, WI 53706-1691 USA (e-mail: nam-heok@cae.wisc.edu).

T. F. Kuech is with the Department of Chemical and Biological Engineering, University of Wisconsin-Madison, Madison, WI 53706-1691 USA.

M. Kanskar is with Alfalight Inc., Madison, WI 53704 USA (e-mail: mkanskar@alfalight.com).

Digital Object Identifier 10.1109/LPT.2006.872283 have been few reports on the temperature sensitivity of the device characteristics using cavity length analysis [3], [4]. A recent experiment [5] has identified carrier leakage out of the QD as an underlying cause for the observed low modal gain and the relatively high temperature sensitivity of self-assembled QD lasers operating near $1 \mu \mathrm{m}$. Studies on longer wavelength QD lasers (near $1.3 \mu \mathrm{m}$ ) indicate Auger recombination also plays a role in the device temperature sensitivity [6]. Carriers which are thermally excited into the wetting layers surrounding the QD, lead to gain saturation at values significantly lower than achievable if saturation occurs due to full population inversion in the dot states. As a result, many stacked layers of QD material are generally needed to achieve typical threshold gain requirements in diode lasers. In addition, this carrier leakage process is highly temperature sensitive.

Recently, the use of a higher energy bandgap matrix such as tensile-strained $\mathrm{GaP}$ matrix [7] embedding the QDs has been investigated, which is expected to improve carrier confinement in the QD, and result in higher peak gain. In this letter, we report a detailed study of the temperature sensitivity of the optical and electrical properties of multiple stacked InGaAs QD lasers grown within a $\mathrm{GaAs}_{0.8} \mathrm{P}_{0.2}$ matrix.

\section{EXPERIMENT}

The InGaAs QD laser structures studied here are grown on nominally exact (100) GaAs substrates by low-pressure (50 mbar) horizontal MOCVD. Laser structures consist of symmetric $1.1-\mu \mathrm{m}$-thick $\mathrm{n}$ - and p-type $\mathrm{Al}_{0.7} \mathrm{Ga}_{0.3} \mathrm{As}$ cladding layers, $0.15-\mu \mathrm{m}$-thick GaAs waveguide layers, and a $0.25-\mu \mathrm{m}$-thick $\mathrm{p}^{+}-\mathrm{GaAs}$ capping layer. Stacked QD layers are sandwiched within the GaAs waveguide layers. Each QD layer is grown on $\mathrm{GaAsP}_{0.2}$ and covered by $\mathrm{GaAsP}_{0.2}$ and the TMIn/total group III flow ratio was 0.6 for the InGaAs QD growth, corresponding to a solid phase In composition of approximately 35\%. Detailed growth conditions for the QD and barrier materials were reported previously [8]. For the threeand five-stack QD structures, a 50-nm GaAs separation layer was grown which is enough distance to uncouple of the strain of the QD layers [9].

The structures were fabricated into conventional broad stripe lasers with $100-\mu \mathrm{m}$-wide oxide defined stripes. The GaAs top contact layer was removed by chemical etching outside of the stripe area before the oxide deposition in order to prevent significant current spreading. Light versus current curves of the as-cleaved devices were measured on a variable temperature stage under pulsed (low duty cycle) conditions up to $2 \mathrm{~A}$. The temperature-dependent characterization of the QD lasers was performed over a range of $10^{\circ} \mathrm{C}-60^{\circ} \mathrm{C}$ with $5^{\circ} \mathrm{C}$ step. 
TABLE I

Threshold CURRENT DENSITY, LASING WAVELENGTH, DIFFERENTIAL QUANTUM EFFICIENCIES FOR THREE- AND FIVE-STACK QD LASERS AT $20^{\circ} \mathrm{C}$

\begin{tabular}{|c|c|c|c|c|}
\hline \multirow{2}{*}{ Barrier } & Cavity Length & $J_{\text {th }}\left(\mathrm{A} / \mathrm{cm}^{2}\right)$ & $\lambda(\mathrm{nm})$ & $\eta_{\mathrm{d}}$ \\
\hline \multirow{2}{*}{$\begin{array}{c}\text { 3-stacked QD } \\
\text { on GaAsP }\end{array}$} & $2 \mathrm{~mm}$ & 214 & 1091 & 58.8 \\
\cline { 2 - 5 } & $3 \mathrm{~mm}$ & 166 & 1097 & 47.9 \\
\cline { 2 - 5 } & $4 \mathrm{~mm}$ & 137 & 1098 & 50.6 \\
\hline \multirow{2}{*}{$\begin{array}{c}\text { 5-stacked QD } \\
\text { on GaAsP }\end{array}$} & $2 \mathrm{~mm}$ & 309 & 1099 & 60.0 \\
\cline { 2 - 5 } & $3 \mathrm{~mm}$ & 228 & 1103 & 55.5 \\
\cline { 2 - 5 } & $4 \mathrm{~mm}$ & 182 & 1110 & 52.8 \\
\hline
\end{tabular}

TABLE II

SUMMARIZED CHARACTERISTIC TEMPERATURES

\begin{tabular}{|c|c|c|c|c|}
\hline structure & $\mathrm{T}_{\text {ini }}(\mathrm{K})$ & $\mathrm{T}_{\mathrm{go}}(\mathrm{K})$ & $\mathrm{T}_{\mathrm{tr}}(\mathrm{K})$ & $\mathrm{T}_{\alpha \mathrm{i}}(\mathrm{K})$ \\
\hline 3-stacked QD & 357 & negative & 79 & 217 \\
\hline 5-stacked QD & 2000 & infinite & 74 & 286 \\
\hline
\end{tabular}

The temperature dependence of the threshold current density (defined by $T_{0}$ ) and the external differential quantum efficiency (defined by $T_{1}$ ) empirically follows the known equations

$$
\begin{aligned}
J_{\mathrm{th}}(T) & =J_{\mathrm{tho}} \cdot e^{\left(T / T_{o}\right)} \\
\eta_{d}(T) & =\eta_{\mathrm{do}} \cdot e^{-\left(T / T_{1}\right)} .
\end{aligned}
$$

Injection efficiencies $\eta_{\text {inj }}$ and internal losses $\alpha_{i}$ were calculated from the measured external differential quantum efficiency versus cavity length. Transparency current densities $J_{\mathrm{tr}}$ and the material gain parameter $g_{0}$ were calculated from measurements of $J_{\mathrm{th}}$ versus cavity length. Temperature-dependent cavity length studies allow for the extraction of the temperature dependence of $J_{\mathrm{tr}}, g_{0}, \eta_{\mathrm{inj}}$, and $\alpha_{i}$ laser parameters. Each laser parameter can then be characterized by a characteristic temperature coefficient $\left(T_{\mathrm{tr}}, T_{g 0}, T_{\eta \mathrm{inj}}, T_{\alpha i}\right)$, defining its exponential dependence on temperature [4]. This type of analysis is useful for achieving a deeper understanding of the overall temperature sensitivity of $J_{\mathrm{th}}$ and $\eta_{d}$, since $T_{0}$ and $T_{1}$ can be expanded in terms of the temperature dependence of each laser parameter as follows:

$$
\begin{aligned}
\frac{1}{T_{o}(L)}= & \frac{1}{T_{\mathrm{tr}}}+\frac{1}{T_{\eta \mathrm{inj}}}+\frac{\alpha_{i}+\alpha_{m}(L)}{\Gamma \cdot g_{o}} \\
& \cdot \frac{1}{T_{g_{o}}}+\frac{\alpha_{i}}{\Gamma \cdot g_{o}} \cdot \frac{1}{T_{\alpha i}} \\
\frac{1}{T_{1}(L)}= & \frac{1}{T_{\eta \mathrm{inj}}}+\frac{\alpha_{i}}{\alpha_{i}+\alpha_{m}(L)} \cdot \frac{1}{T_{\alpha i}} .
\end{aligned}
$$

\section{RESULTS AND DISCUSSION}

The threshold current density $\left(J_{\mathrm{th}}\right)$, lasing wavelength, and differential quantum efficiency $\left(\eta_{d}\right)$ of $2000-4000-\mu$ m cavity length devices at room temperature are summarized in Table I. The extracted $T_{\mathrm{tr}}, T_{g 0}, T_{\eta \mathrm{inj}}, T_{\alpha i}$ are shown in Table II.

The room temperature $J_{\mathrm{th}}$ values for the five-stack QD structure are $\sim 1.3$ times higher than three-stack QD structure. While the transparency current density should increase with active layer volume, we also find the material gain coefficient $g_{0}$ is lower for the five-stack devices, leading to an increased threshold current density. Additional thermal annealing of the

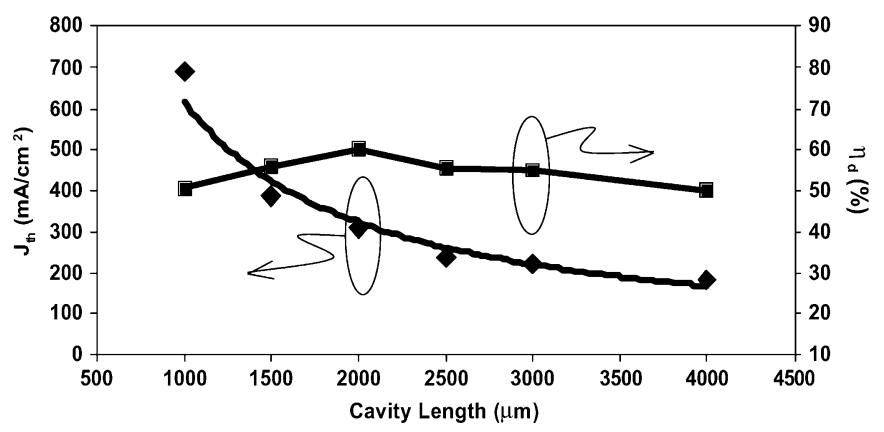

Fig. 1. Threshold current density and external differential quantum efficiency as a function of cavity length $L$.

lower QD stacks during the growth of the upper QD stacks may impact the optical properties, resulting in the observed lower material gain coefficient. Extracted $g_{0}$ values from five-stack QD devices at $20{ }^{\circ} \mathrm{C}$ were $75 \%$ that of the three-stack $g_{0}$ values. Optical confinement factors $(\Gamma)$ of 0.0161 and 0.0217 were calculated for the three- and five-stack structures, respectively, allowing extraction of $g_{0}$ from the threshold condition $\Gamma \cdot g_{\mathrm{th}}=\alpha_{i}+\alpha_{m}$, where $g_{\mathrm{th}}=g_{0} \cdot \operatorname{Ln}\left(\eta_{\mathrm{inj}} \cdot J_{\mathrm{th}} / J_{\mathrm{tr}}\right)$. A larger confinement factor for the five-stack structure will result in a lower threshold carrier density (assuming same internal loss) compared to the three-stack structure. This may be partially responsible for the lower gain parameter observed for the five-stack structure (i.e., less contribution of gain from excited states). Other reasons for lower gain may be from variations in QD properties among the stacks. Further studies are necessary to fully elucidate the reasons for the lower gain parameter observed for the five-stack devices. The extraction of the material gain parameter from multiple stack QD lasers is found to be a sensitive tool to quantify the material quality for increasing number of QD stacks, and will allow us to optimize growth procedures.

Fig. 1 shows the dependences of the $J_{\text {th }}$ and $\eta_{d}$ as a function of cavity length for lasers with a five-stack QD structure. For all cavity length studies, the calculated values were extracted from 2000- to 4000- $\mu \mathrm{m}$ devices, to avoid excessive band filling or lasing on excited QD states found in shorter cavity length devices. The emission wavelength increases continuously with temperature for the three-stack QD devices, indicating lasing on the ground state transition throughout the temperature range. The differential emission wavelength changes with the temperature $(\Delta \lambda / \Delta T)$ are $0.21-0.24 \mathrm{~nm} /{ }^{\circ} \mathrm{C}$ for $2000-4000-\mu \mathrm{m}$ devices. Ground state lasing is dominant for long cavity devices, although contributions from excited state gain contribute at shorter cavity lengths and higher temperatures, resulting in a reduced wavelength temperature sensitivity [10].

The $T_{0}$ values of all the chips are lower than $100 \mathrm{~K}$, which is generally lower than that of conventional InGaAs quantum-well (QW) devices (120-150 K) with similar transverse structures. The lower $T_{0}$ values observed for the QD lasers may result from increasing carrier leakage at elevated temperatures through excited or wetting layer states [5]. As shown in (3), $T_{0}$ values can be expressed as a function of characteristic temperature coefficients $\left(T_{\mathrm{tr}}, T_{g 0}, T_{\eta \mathrm{inj}}\right.$, and $\left.T_{\alpha i}\right)$. We find the calculated $T_{g 0}, T_{\eta \mathrm{inj}}, T_{\alpha i}$ values are reasonably high (characteristic temperature coefficients will be discussed later), and that the low $T_{0}$ values of the QD lasers correlate with a low value of $T_{\mathrm{tr}}$. 


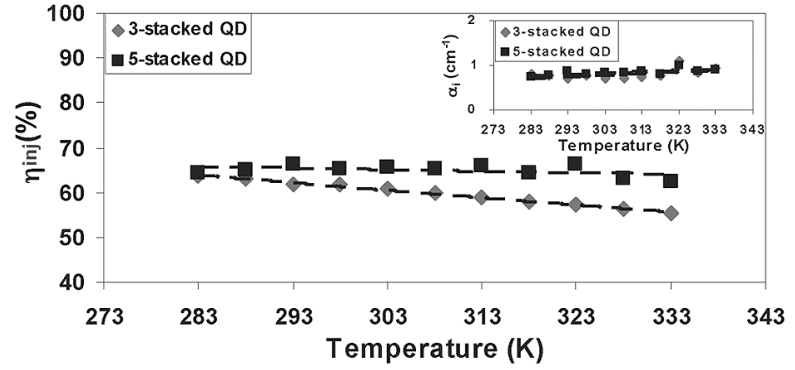

Fig. 2. Current injection efficiency as a function of temperature for threeand five-stacked QD structure. Inset figure shows internal loss as a function of temperature for three- and five-stacked QD structure.

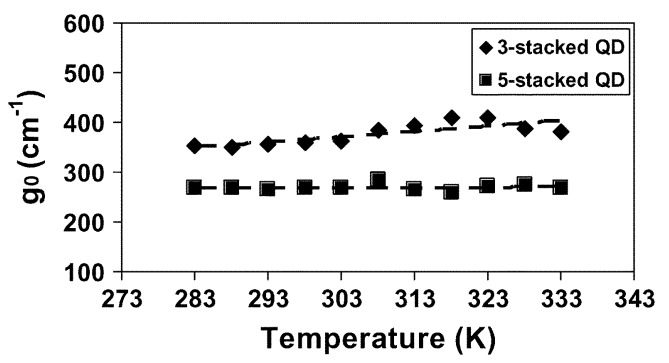

Fig. 3. Material gain as a function of temperature for three- and five-stack QD structure.

The calculated $T_{1}$ values of the three- and five-stack QD devices are reasonably high $(>230 \mathrm{~K}$ for three-stacks, $>400 \mathrm{~K}$ for five-stacks). From (4), $T_{1}$ values can be expressed as a function of $T_{\eta \text { inj }}$ and $T_{\alpha i}$. We find the high $T_{1}$ values correlate with low temperature sensitivity of the injection efficiency and internal loss (i.e., $T_{\eta \text { inj }}, T_{\alpha i}$ values are high).

The temperature-dependent injection efficiency $\left(\eta_{\text {inj }}\right)$ and internal loss $\left(\alpha_{i}\right)$ were calculated from the measured $\eta_{d}$ with various cavity lengths, as shown Fig. 2 . We find the $\eta_{\text {inj }}$ values are significantly lower than conventional InGaAs QW lasers (90\%-95\%). The low growth temperature of the QD cover layer may introduce a large amount of nonradiative recombination centers into the material surrounding the QDs [2]. The injection efficiency is expected to be reduced due to monomolecular recombination outside the QDs. Optimized annealing conditions have been found by Sellin et al. to strongly effect the laser characteristics, possibly due to a reduction of the defect density near the QD and GaAs spacer layers [2]. Similar optimization is expected to improve the material quality of the GaAsP matrix in our device structures. The five-stack QD structure exhibits a high characteristic temperature coefficient of the injection efficiency $\left(T_{\eta \text { inj }}\right)$, leading to a high $T_{1}$ value. Extracted $\alpha_{i}$ values for all the devices over the entire temperature range are near or less than $1 \mathrm{~cm}^{-1}$ and characteristic temperature coefficient of the internal loss $\left(T_{\alpha i}\right)$ is higher than $200 \mathrm{~K}$.

Fig. 3 shows the extracted material gain $\left(g_{0}\right)$ parameter as a function of temperature. The five-stack QD laser structure has a lower gain parameter than the three-stack structure, which directly leads to a higher threshold current density. Surprisingly, we find the material gain values increase with increasing temperature for the three-stack QD structure, and are nearly temperature independent for the five-stack structure. This behavior has not been reported for InGaAs QW lasers, which exhibit a decreasing $g_{0}$ with increasing temperature [11]. The redistribu- tion of carriers between ground and excited states [10] may be responsible for the observed $g_{0}(T)$ behavior in these devices. At higher temperatures, the excited states may contribute additional gain, resulting in a negative or nearly constant slope of the temperature dependence of the material gain.

\section{SUMMARY}

InGaAs QD lasers in the 1- $\mu$ m wavelength region exhibit low $J_{\text {th }}$ values $\left(100 \times 4000 \mu \mathrm{m}\right.$, as-cleaved) of $137 \mathrm{~A} / \mathrm{cm}^{2}$ for threestack and $182 \mathrm{~A} / \mathrm{cm}^{2}$ for five-stack devices at room temperature, respectively. The $T_{1}$ values of three-stack QD laser structures are higher than $400 \mathrm{~K}$. On the contrary, $T_{0}$ values are lower than $100 \mathrm{~K}$, significantly lower than comparable QW lasers.

Temperature-dependent cavity length analysis indicates that the high $T_{1}$ and low $T_{0}$ values for the QD lasers originate from low temperature sensitivity of both the injection efficiency and internal loss (i.e., high $T_{\eta \text { inj }}, T_{\alpha i}$ ) and the high temperature sensitivity of the transparency current density (i.e., low $T_{\mathrm{tr}}$ ). Extracted material gain values increase with increasing temperature for the three-stack QD structure, and are nearly temperature independent for the five-stack structure, very different from the behavior of InGaAs QW lasers. Optimization of the GaAsP material surrounding the InGaAs QDs is expected to yield further improvement in device characteristics.

\section{REFERENCES}

[1] Y. Arakawa and H. Sakaki, "Multidimensional quantum well laser and temperature dependence of its threshold current," Appl. Phys. Lett., vol. 40, pp. 939-941, 1982.

[2] R. L. Sellin, Ch. Ribbat, M. Grundmann, N. N. Ledentsov, and D. Bimberg, "Close-to-ideal device characteristics of high-power InGaAs/GaAs quantum dot lasers," Appl. Phys. Lett., vol. 78, pp. 1207-1209, 2001.

[3] F. Klopf, J. P. Reithmaier, and A. Forchel, "Highly efficient $\mathrm{GaInAs} /(\mathrm{Al}) \mathrm{GaAs}$ quantum-dot lasers based on a single active layer versus $980 \mathrm{~nm}$ high-power quantum-well lasers," Appl. Phys. Lett., vol. 77, pp. 1419-1421, 2000.

[4] N. Tansu, Y.-L. Chang, T. Takeuchi, D. P. Bour, S. W. Corzine, M. R. T. Tan, and L. J. Mawst, "Temperature analysis and characteristics of highly strained InGaAs-GaAsP-GaAs $(>1.17 \mu \mathrm{m})$ quantum-well lasers," IEEE Trans. Quantum Electron., vol. 38, no. 6, pp. 640-651, Jun. 2002.

[5] D. R. Matthews, H. D. Summers, P. M. Smowton, and M. Hopkinson, "Experimental investigation of the effect of wetting-layer states on the gain-Current characteristic of quantum-dot lasers," Appl. Phys. Lett., vol. 81, pp. 4904-4906, 2002.

[6] I. P. Marko, A. D. Andreev, A. R. Adams, R. Krebs, J. P. Reithmaier, and A. Forchel, "The role of auger recombination in InAs $1.3-\mu \mathrm{m}$ quantum-dot lasers investigated using high hydrostatic pressure," IEEE J. Sel. Topics Quantum Electron., vol. 9, no. 5, pp. 1300-1307, Sep./Oct. 2003.

[7] P. Lever, H. H. Tan, and C. Jagadish, "InGaAs quantum dots grown with GaP strain compensation layers," J. Appl. Phys., vol. 95, pp. 5710-5714, 2004.

[8] N. H. Kim, P. Ramamurthy, L. J. Mawst, T. F. Kuech, P. Modak, T. J. Goodnough, D. V. Forbes, and M. Kanskar, "Characteristics of InGaAs quantum dots grown on tensile-strained $\mathrm{GaAs}_{1-x} \mathrm{P}_{x}$, J. Appl. Phys., vol. 97, p. $093518,2005$.

[9] M. Gutierrez, M. Hopkinson, H. Y. Liu, A. I. Tartakovskii, M. Herrera, D. Gonzalez, and R. Garcia, "Critical barrier thickness for the formation of InGaAs/GaAs quantum dots," Mater. Sci. Eng. C, vol. 25, pp. 798-803, 2005.

[10] F. Klopf, S. Deubert, J. P. Reithmaier, and A. Forchel, "Correlation between the gain profile and the temperature-induced shift in wavelength of quantum-dot lasers," Appl. Phys. Lett., vol. 81, pp. 217-219, 2002.

[11] N. Tansu and L. J. Mawst, "Temperature sensitivity of 1300-nm InGaAsN quantum-well lasers," IEEE Photon. Technol. Lett., vol. 14, no. 8, pp. 1052-1054, Aug. 2002. 